\title{
Evaluación de resultados de una encuesta de satisfacción del proceso editorial, Revista Médica Hondureña, 2016-2020
}

\author{
Evaluation of results of a satisfaction survey of the editorial process, Revista Médica Hondureña, 2016-2020
}

Edna Maradiaga ${ }^{1-3}$ @ https://orcid.org/0000-0002-8424-4752, Briana Beltran 1,2 — https://orcid.org/0000-0003-0362-4647, Nora Rodríguez Corea ${ }^{1,4}$ @ https://orcid.org/0000-0003-1749-4556, Rosa María Duarte, ${ }^{1,5}$ Chttps://orcid.org/0000-0003-2373-6574, Eleonora Espinoza ${ }^{1,3}$ —D https://orcid.org/0000-0001-7218-3481, Flor Mejía, ${ }^{1,2}$ — https://orcid.org/0000-0002-3033-2722, Ana Ligia Chinchilla, ${ }^{1,6}$ - https://orcid.org/0000-0001-9963-6575, Heriberto Rodríguez ${ }^{1,7}$ ○ https://orcid.org/0000-0003-0333-4201, Melissa Mejía ${ }^{1,2,8}$ @ https://orcid.org/0000-0002-6634-8419, Jackeline Alger ${ }^{1,3,9}$ @ https://orcid.org/0000-0001-9244-0668.

${ }^{1}$ Colegio Médico de Honduras, Revista Médica Hondureña, Consejo Editorial (CE-RMH); Tegucigalpa, Honduras ${ }^{2}$ Colegio Médico de Honduras, Centro Nacional de Educación Médica Continua (CENEMEC); Tegucigalpa, Honduras.

${ }^{3}$ Universidad Nacional Autónoma de Honduras (UNAH), Facultad de Ciencias Médicas (FCM), Unidad de Investigación Científica (UIC), Tegucigalpa, Honduras. ${ }^{4}$ UNAH, FCM, Departamento de Salud Pública, Posgrado en Salud Pública; Tegucigalpa, Honduras.

${ }^{5}$ Secretaría de Salud, Dirección General de Normalización; Tegucigalpa, Honduras.

${ }^{6}$ Instituto Hondureño de Seguridad Social (IHSS), Departamento de Ginecología y Obstetricia; UNAH FCM Departamento de Ciencias Morfológicas; Tegucigalpa, Honduras.

${ }^{7}$ Secretaría de Salud, Hospital de Especialidades San Felipe; Tegucigalpa, Honduras.

${ }^{8}$ Colegio Médico de Honduras, Secretaría de Asuntos Educativos y Culturales; Tegucigalpa, Honduras.

${ }^{9}$ Secretaría de Salud, Hospital Escuela, Departamento de Laboratorio Clínico, Servicio de Parasitología; Tegucigalpa, Honduras.

RESUMEN. Antecedentes: En el proceso editorial participan autores, editores, revisores pares y lectores, quienes contribuyen a la calidad de las publicaciones biomédicas. Objetivo: Evaluar los resultados de una encuesta de satisfacción dirigida a autores y revisores pares que participaron en el proceso editorial de la Revista Médica Hondureña (RMH), periodo 20162020. Metodología: Análisis retrospectivo de los formularios completados en línea por autores y revisores pares, invitados a participar voluntariamente por correo electrónico durante diciembre 2020-julio 2021. La encuesta incluyó 16 preguntas que registraron características del proceso editorial y sugerencias de mejora. La satisfacción se clasificó en 5 categorías, de muy satisfecho a muy insatisfecho. Los resultados se presentan como frecuencias y porcentajes de las variables estudiadas. Resultados: Se analizaron 53 encuestas, completadas por $88.7 \%$ (47) autores y $11.3 \%$ (6) revisores pares. El $94.3 \%$ (50) describió como claras las instrucciones para autores, $77.4 \%$ (41) indicó haber recibido respuesta oportuna, $71.7 \%$ (38) recibió asesoría personalizada, $90.5 \%$ (48) describió como muy probable/probable su disposición a enviar artículos o colaborar como revisor par; algunas limitantes para participar fueron falta de tiempo e inexperiencia. El $69.8 \%$ (37) expresó estar muy satisfecho/satisfecho en comparación a $17.0 \%$ (9) que expresó insatisfacción con el proceso editorial. Discusión: En esta muestra limitada de usuarios, más de $2 / 3$ expresó una percepción positiva y satisfacción con el proceso editorial. Es necesario impulsar la mejora continua de la RMH y promover el fortalecimiento del ecosistema de la investigación en el gremio médico y colaboradores en Honduras.

Palabras clave: Autoría y coautoría en la publicación científica; Políticas editoriales; Publicación académica; Revisión por pares; Satisfacción personal.

\section{INTRODUCCIÓN}

El proceso editorial es una secuencia de pasos que inicia con el envío de un manuscrito "el producto de una investigación" hasta el ejemplar ya publicado (Figura 1). Este proceso implica el involucramiento de muchos actores, entre los cuales podemos mencionar los autores, los editores, los revisores externos y por supuesto los lectores. ${ }^{1}$ La actualización de los conocimientos y las habilidades de los autores, editores, el desarrollo y la aprobación de recomendaciones de asociaciones editoriales mundiales y la redacción de las instrucciones de las revistas pueden considerarse herramientas potenciales para mejorar la ética y la calidad de las revistas académicas. ${ }^{2}$

Un buen proceso editorial también lleva consigo muchos procesos operativos regidos por altos estándares de calidad nacionales e internacionales. La Revista Médica Hondureña $(\mathrm{RMH})$ ha tenido un proceso de renovación editorial, que ha sido constante durante varios años. Sin embargo, desde el 2019 se ha dado un impulso al fortalecimiento y mejora de éste, con

Recibido: 17-11-2021 Aceptado: 13-12-2021 Primera vez publicado en línea: 17-12-2021 Dirigir correspondencia a: Dra. Edna Maradiaga Correo electrónico: edjamar3006@yahoo.com

DECLARACIÓN DE RELACIONES Y ACTIVIDADES FINANCIERAS Y NO FINANCIERAS: Ninguna.

DECLARACIÓN DE CONFLICTOS DE INTERÉS: Ninguno.

Forma de citar: Maradiaga E, Beltran B, Rodríguez Corea N, Duarte RM, Espinoza E, Mejía F, Chinchilla AL, Rodríguez H, Mejía M, Alger J. Evaluación de resultados de una encuesta de satisfacción del proceso editorial, Revista Médica Hondureña, 2016-2020. Rev Méd Hondur. 2021; 89 (2): 124-130. DOI: https://doi.org/10.5377/rmh.v89i2.13020

(C) 2021 Autor(es). Artículo de acceso abierto bajo la licencia https://creativecommons.org/ licenses/by/4.0/deed.es

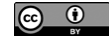




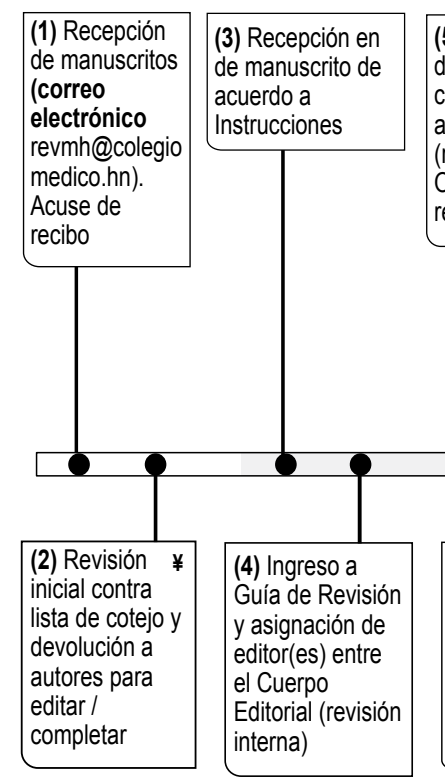

(5) Revisión interna $¥ \S$ de formato y contenido completada. Se asigna a revisor(es) par (revisión externa. Original, mínimo dos revisores par

(

$¥$ Retorno a autor(es), § Tutoría presencial y/o virtual

* LILACS lilacs.bvsalud.org/es/, CAMJOL www.camjol.info/index.php/RMH, AMELICA http://portal.amelica.org/revista.oa?id=470, LATINDEX https://www.latindex.org/latindex/ficha?folio=11879

(7) Revisión por $\quad ¥$ BIMENA completada. Envío a diagramación. Se informa a autores (correo electrónico $\mathrm{RMH} 2)$

\section{(9) Gestión de} registro en plataforma

CAMJOL para obtención del identificador DOI (BIMENA)
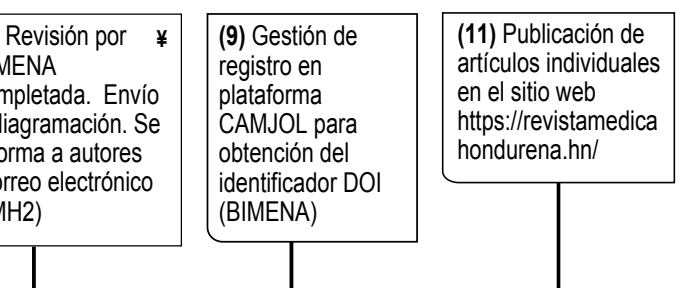

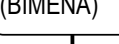

(13) Envío de documento completo a BIMENA para registro en Biblioteca

Virtual en Salud de

Honduras

(www.bvs.hn/RMH). Se completa registro en todas las plataformas internacionales (CAMJOL, AMELICA, LILACS, LATINDEX, otras)*

Figura 1. Flujo de los manuscritos sometidos al proceso editorial de la Revista Médica Hondureña, Honduras.

énfasis en los parámetros bibliométricos, uso de estándares de publicación y la sistematización del proceso de revisión por pares. Se ha reconocido en el ambiente académico que el sistema de revisión por pares ha permitido el control de calidad de los manuscritos enviados para su publicación en revistas científicas. $^{3,4}$ Las mejoras implementadas por la RMH van desde, el fortalecimiento del consejo editorial, trazabilidad de procesos, puntualidad, establecimiento de parámetros bibliométricos, con la finalidad de poder alcanzar la indización en diferentes bases de datos internacionales.

Para comprender los aspectos de mejora del proceso editorial, es fundamental tener claridad de los indicadores de calidad de las revistas científicas. Estos indicadores los podemos dividir en indicadores de estructura, de procesos y de resultados. Los indicadores de estructura se refieren al cumplimiento de las normas de los procesos editoriales, los indicadores de proceso a los relacionados con la gestión, visibilidad y arbitraje editorial. Entre los indicadores de resultados tenemos el número de artículos descargados al estar en una base de datos en internet, pudiéndose obtener por mes, año y a su vez el índice de citación de los artículos publicados en las revistas, entre los que se consideran el factor de impacto y el índice de inmediatez utilizado por Thomson Scientific y SciELO. ${ }^{5}$

Según el análisis bibliométrico realizado por la RMH comprendido desde 1990-2020, se dio a conocer que la producción científica de la RMH es diversa de acuerdo con su alcance, pero escasa en cantidad y visibilidad. Es necesario incorporar más indicadores bibliométricos para su indización en bases de datos internacionales y plataformas, incluyendo las redes sociales, para aumentar su impacto y visibilidad. ${ }^{6}$ Para mantener la ca- lidad en todos los procesos editoriales de las revistas, aparte de tener presente los indicadores antes mencionados, es de mucha importancia contar con la opinión de autores y revisores pares que han participado en el proceso editorial, lo cual contribuye a la mejora continua de la revista. Según lo antes expuesto, el presente estudio se realizó con el objetivo de evaluar los resultados de una encuesta de satisfacción dirigida a autores y revisores pares que participaron en el proceso editorial de la RMH en un periodo de cinco años, del 2016 al 2020.

\section{MATERIALES Y MÉTODOS}

Se llevó a cabo un análisis retrospectivo de los formularios completados en línea durante diciembre 2020 a julio 2021 por usuarios autores y revisores pares de la RMH. Los usuarios fueron identificados por el Cuerpo Editorial a partir de la base de datos de autores y revisores pares del periodo 2016 a 2020 y fueron invitados a participar voluntariamente por correo electrónico. En la invitación a participar se explicó que se trataba de una encuesta auto administrada sobre el último proceso editorial que había experimentado, bajo un manejo anonimizado de la información personal de los participantes.

El formulario en línea consistió en 16 preguntas que registraron características del usuario (autor, revisor) y tiempo de la experiencia (2016-2020) (2 preguntas), conocimiento del sitio web (3 preguntas, 2 enlazadas a respuesta positiva), sobre la claridad, oportunidad y efectividad de las instrucciones y comunicación en el proceso editorial (5 preguntas, 1 con explicación de razones), participación en asesoría y medio de participación (2 preguntas), satisfacción general y posibilidad 
de participar nuevamente ( 3 preguntas) y sugerencias de mejora (1 pregunta). La satisfacción general con el proceso editorial se clasificó en 5 categorías incluyendo muy satisfecho, satisfecho, neutral, insatisfecho y muy insatisfecho. Este instrumento de recolección fue elaborado por miembros del Cuerpo Editorial de la RMH.

Los datos obtenidos en la encuesta se interpretaron en un análisis descriptivo a través de frecuencias y porcentajes de las variables estudiadas. Para realizar este análisis, se contó con el aval institucional de la RMH y del Centro Nacional de Educación Médica Continua (CENEMEC) del Colegio Médico de Honduras.

\section{RESULTADOS}

De un total de 125 invitados, $87.2 \%$ (109) fueron autores y $12.8 \%$ (16) revisores pares, participaron 53 usuarios, $88.7 \%$ (47) como autor y $11.3 \%$ (6) como revisor par. En relación con el año de su experiencia más reciente de un proceso editorial, $50.9 \%$ (27) fue en 2020 y un $20.8 \%$ (11) en 2019. Con respecto al conocimiento del sitio web de la $\mathrm{RMH}, 84.9 \%$ (45) respondió que sí tenía conocimiento y de éstos $23 / 45$ lo habían visitado entre 2 a 5 veces en los últimos dos meses. Además, $56.6 \%$ (30) lo había compartido con otros colegas y estudiantes (Cuadro 1).

Entre las características del proceso editorial exploradas, $94.3 \%$ (50) manifestó que las instrucciones para autores eran claras. El $77.4 \%$ (41) indicó haber recibido respuesta oportuna al enviar el manuscrito vía correo electrónico. En relación con

Cuadro 1. Características del usuario, tiempo de la experiencia y conocimiento del sitio web de la Revista Médica Hondureña (RMH), Honduras, 2016-2020, n=53.

\begin{tabular}{lcc}
\hline & $\mathbf{n}$ & $(\%)$ \\
\hline Mi última experiencia en un proceso editorial de la RMH & & \\
Autor & 47 & $(88.7)$ \\
Revisor par & 6 & $(11.3)$ \\
Esa última experiencia fue en el año: & & \\
2016 & 4 & $(7.5)$ \\
2017 & 5 & $(9.4)$ \\
2018 & 6 & $(11.3)$ \\
2019 & 11 & $(20.7)$ \\
2020 & 27 & $(50.9)$ \\
Conocimiento del sitio web de la RMH & & \\
No & 8 & $(15.1)$ \\
Sí & 45 & $(84.9)$ \\
Visitas al sitio web en los 2 últimos meses. & & \\
1 & 15 & $(33.3)$ \\
$2-3$ & 15 & $(33.3)$ \\
$4-5$ & 8 & $(17.8)$ \\
$>5$ & 7 & $(15.6)$ \\
Compartido sitio web con colegas/estudiantes & & \\
Sí & 30 & $(56.6)$ \\
No & 19 & $(35.8)$ \\
NA & 4 & $(7.5)$ \\
\hline
\end{tabular}

NA= No aplica las respuestas enviadas por el Consejo Editorial, 24.5\% (13) expresó que eran extremadamente claras y $52.8 \%$ (28) muy claras. En cuanto al nivel de información que se les proporcionó a los usuarios sobre el proceso de revisión del manuscrito, $22.6 \%$ (12) reportó estar muy informado y $52.8 \%$ (28) informado adecuadamente. En cuanto al nivel de efectividad del cumplimiento del tiempo en cada uno de los pasos del proceso editorial, $26.4 \%$ (14) informó ser muy efectivo y $47.2 \%$ (25) que había tenido una efectividad adecuada. El 71.7\% (38) recibió asesoría personalizada para aclarar dudas con relación a la revisión de su manuscrito y los medios utilizados fueron $71.6 \%$ (38) vía WhatsApp y $69.8 \%$ (37) vía correo electrónico (Cuadro 2).

Cuadro 2. Claridad, oportunidad y efectividad del proceso editorial mediante instrucciones, comunicación y asesoría, Revista Médica Hondureña (RMH), 2016-2020, $n=53$.

\begin{tabular}{|c|c|c|}
\hline & $\mathrm{n}$ & $(\%)$ \\
\hline \multicolumn{3}{|c|}{ Instrucciones para autores son claras. } \\
\hline $\mathrm{Si}$ & 50 & (94.3) \\
\hline No & 3 & $(5.6)$ \\
\hline \multicolumn{3}{|c|}{$\begin{array}{l}\text { Respuesta oportuna al enviar su manuscrito por } \\
\text { correo electrónico. }\end{array}$} \\
\hline $\mathrm{Si}$ & 41 & $(77.4)$ \\
\hline No & 11 & $(20.7)$ \\
\hline NC & 1 & $(1.9)$ \\
\hline \multicolumn{3}{|c|}{ Claridad de las respuestas que se envían. } \\
\hline Extremadamente claras & 13 & $(24.5)$ \\
\hline Muy claras & 28 & $(52.8)$ \\
\hline Moderadamente claras & 1 & $(1.9)$ \\
\hline Un poco claras & 10 & (18.8) \\
\hline Nada claras & 1 & $(1.9)$ \\
\hline \multicolumn{3}{|c|}{$\begin{array}{l}\text { Información sobre el proceso de su artículo o } \\
\text { revisión de manuscrito }\end{array}$} \\
\hline Muy informado & 12 & $(22.6)$ \\
\hline Informado adecuadamente & 28 & $(52.8)$ \\
\hline Poco informado & 12 & (22.6) \\
\hline Nada informado. & 1 & $(1.9)$ \\
\hline \multicolumn{3}{|c|}{$\begin{array}{l}\text { Nivel de efectividad del cumplimiento con el } \\
\text { tiempo en cada paso del proceso editorial. }\end{array}$} \\
\hline Muy efectivo & 14 & (26.4) \\
\hline Efectividad adecuada & 25 & $(47.2)$ \\
\hline Poco efectivo & 13 & (24.5) \\
\hline Nada efectivo & 1 & $(1.9)$ \\
\hline \multicolumn{3}{|c|}{$\begin{array}{l}\text { Recibió asesoría personalizada para aclarar } \\
\text { dudas revisión de su manuscrito. }\end{array}$} \\
\hline $\mathrm{Si}$ & 38 & $(71.7)$ \\
\hline No & 12 & (22.6) \\
\hline NA & 3 & $(5.6)$ \\
\hline \multicolumn{3}{|c|}{ Medios utilizados para la asesoría* } \\
\hline Vía WhatsApp & 38 & $(71.6)$ \\
\hline Vía correo electrónico & 37 & $(69.8)$ \\
\hline Vía teléfono & 7 & (13.2) \\
\hline Vía Zoom & 6 & (11.3) \\
\hline Presencial & 1 & $(1.9)$ \\
\hline
\end{tabular}

$\mathrm{NC}=$ No contesta, $\mathrm{NA}=$ No aplica 
Con respecto al nivel de satisfacción del proceso editorial al someter los manuscritos planteado por los usuarios, se encontró que $41.5 \%$ (22) estaba muy satisfecho, $28.3 \%$ (15) satisfecho y $17.0 \%$ (9) que expresó insatisfacción. El 56.6\% (30) de los usuarios expresó como muy probable su disposición para enviar artículos nuevamente o colaborar como revisor par, reflejándose más en el año 2020. Entre las limitaciones que expresaron los autores para enviar nuevamente un manuscrito para publicación, se encontró lo siguiente: no tiene experiencia $86.8 \%$ (46), desconoce cómo hacerlo $86.8 \%$ (46), no cuenta con un equipo de trabajo $77.4 \%$ (41). Los revisores pares expresaron que no cuentan con experiencia 11.3\% (6) (Cuadro 3).

\section{DISCUSIÓN}

En esta muestra de usuarios autores y revisores pares de los últimos cinco años de la RMH, se identificó un nivel de aproximadamente $70 \%$ de satisfacción que habían experimentado con el proceso editorial. La RMH está integrada a las actividades de educación médica continua del Colegio Médico de Honduras y considera importante conocer cómo perciben los usuarios la calidad del servicio o producto brindado ya que esa información contribuye a la implementación de mejoras. En re- lación con los usuarios, E. Deming introduce el concepto que "la calidad se define en términos de quien la valora", es decir el usuario, apareciendo el concepto del control total de la calidad, es decir calidad del producto, calidad del proceso y la satisfacción del cliente. ${ }^{5} \mathrm{Si}$ aplicamos estos conceptos a las revistas científicas, el producto sería la publicación impresa o electrónica; los procesos se refieren al proceso editorial y al proceso administrativo; y los usuarios a los que hay que satisfacer son los lectores, investigadores, profesionales e instituciones. En este sentido es importante conocer cómo perciben los usuarios, la calidad de un servicio o producto. Así mismo, Weber et al aborda el "Impacto de la calidad y aceptación de la revisión en la satisfacción", indica que la satisfacción de los usuarios estuvo asociada con la aceptación del manuscrito. ${ }^{7}$ En el caso de la RMH es muy probable que la satisfacción de los usuarios, en especial de los autores se pueda relacionar también con la aceptación del manuscrito y las mejoras del proceso editorial recientes.

Por otra parte, se encontró que $17.0 \%$ (9) refirió insatisfacción del proceso editorial de la $\mathrm{RMH}$, si bien es cierto es un porcentaje bajo debe llamar a la reflexión al cuerpo editorial de la revista e indagar a futuro los factores que contribuyen a esta situación y así mejorar este aspecto. Según algunos es-

Cuadro 3. Satisfacción del proceso editorial y posibilidad de participar de nuevo según el período de la experiencia, Revista Médica Hondureña (RMH), 2016-2020, n=53.

\begin{tabular}{|c|c|c|c|c|c|c|}
\hline & $\begin{array}{c}2016 \\
n=4\end{array}$ & $\begin{array}{c}2017 \\
n=5\end{array}$ & $\begin{array}{c}2018 \\
n=6\end{array}$ & $\begin{array}{l}2019 \\
n=11\end{array}$ & $\begin{array}{l}2020 \\
n=27\end{array}$ & Total \\
\hline & $\mathbf{n}$ & $\mathrm{n}$ & $\mathrm{n}$ & $\mathbf{n}$ & $\mathrm{n}$ & $\mathrm{n}(\%)$ \\
\hline \multicolumn{7}{|c|}{ Nivel de satisfacción con el proceso editorial: } \\
\hline Muy satisfecho & - & - & 1 & 2 & 19 & $22(41.5)$ \\
\hline Satisfecho & 1 & 1 & 2 & 7 & 4 & $15(28.3)$ \\
\hline Neutral & 1 & 3 & & 1 & 1 & $6(11.3)$ \\
\hline Insatisfecho & 2 & 1 & 2 & 1 & 3 & $9(17.0)$ \\
\hline NC & & & 1 & & & $1(1.9)$ \\
\hline \multicolumn{7}{|c|}{$\begin{array}{l}\text { Probabilidad de que envíe nuevamente a la RMH un artículo } \\
\text { para publicar o colabore como revisor par. }\end{array}$} \\
\hline Muy probable & 1 & 1 & 2 & 6 & 20 & $30(56.6)$ \\
\hline Probable & 2 & 3 & 3 & 4 & 6 & $18(33.9)$ \\
\hline Poco probable & 1 & 1 & 1 & 1 & 1 & $5(9.4)$ \\
\hline \multicolumn{7}{|c|}{$\begin{array}{l}\text { Limitantes actuales para poder colaborar nuevamente con } \\
\text { la RMH: }\end{array}$} \\
\hline \multicolumn{7}{|c|}{ Autor* } \\
\hline -No sé cómo hacerlo & 3 & 5 & 6 & 11 & 21 & $46(86.8)$ \\
\hline -No tengo experiencia & 3 & 5 & 6 & 10 & 22 & $46(86.8)$ \\
\hline -No cuento con un equipo de trabajo & 2 & 5 & 6 & 10 & 18 & $41(77.4)$ \\
\hline -No cuento con información original & 2 & 4 & 4 & 9 & 21 & $40(75.5)$ \\
\hline -Falta de tiempo & 1 & - & 2 & 5 & 9 & $17(32.1)$ \\
\hline \multicolumn{7}{|l|}{ Revisor par* } \\
\hline -No cuento con información original & 1 & - & - & - & 5 & $6(11.3)$ \\
\hline -No sé cómo hacerlo & 1 & - & - & - & 5 & $6(11.3)$ \\
\hline -No tengo experiencia & 1 & - & - & - & 5 & $6(11.3)$ \\
\hline -No cuento con un equipo de trabajo & 1 & - & - & - & 5 & $6(11.3)$ \\
\hline -Falta de tiempo & 1 & - & - & - & 2 & $3(5.7)$ \\
\hline
\end{tabular}

*Los participantes seleccionaron más de una opción $\mathrm{NC}=$ No contesta 
tudios relacionados con las perspectivas del autor, mencionan que, si los editores se toman mucho tiempo para un rechazo del manuscrito, es probable que también demoren más tiempo en encontrar revisores y procesar los informes de los revisores entrantes. Por lo tanto, el tiempo de rechazo inmediato es un indicador poderoso del desempeño general de las oficinas editoriales pudiendo influenciar el nivel de satisfacción de los usuarios. $^{8}$

Con respecto al nivel de efectividad del cumplimiento con el tiempo en cada paso del proceso editorial se encontró que el $73.6 \%$ reportó entre muy eficaz y efectividad adecuada y solo el $24.5 \%$ refirió poca efectividad. Según estos resultados, se podría entender que el trabajo dedicado de los editores ha desempeñado un papel importante. Sin embargo, todavía hay retos de mejora continua como los tiempos de cumplimiento, que depende de los tiempos los revisores, editores y autores, es decir, que lleva consigo un engranaje complejo y multidisciplinario. Por otro lado, el tiempo total entre el envío de un manuscrito y la decisión final del editor no solo está influenciado por el tiempo que los revisores y el tiempo que las oficinas editoriales tardan en manejar el manuscrito, sino también por el tiempo que tardan los autores en revisar y volver a enviar el artículo. ${ }^{8}$ Los tiempos de manipulación más cortos también influyen positivamente en la reputación y el nivel de satisfacción siempre que indiquen una manipulación de manuscritos rápida y fiable. ${ }^{9}$

La mitad de los entrevistados (autores y revisores pares) expresaron que su experiencia era reciente, entre uno y dos años, $79 \%$ (42) de los participantes manifestaron que las instrucciones para los autores de la RMH eran claras. Para la presentación del artículo las revistas pueden brindar a los autores una lista de comprobación para que de forma rápida comprueben que cumplen los requisitos en cada apartado. Los autores pueden ayudarse de las listas guías de comprobación publicadas para cada tipo de informe de investigación disponibles en EQUATOR (http://www.equator-network.org). ${ }^{10,11}$ Las pautas antes descritas se encuentran entre los recursos para autores, en donde también se comparte plantillas de los diferentes tipos de artículos las cuales periódicamente se van actualizando, esto con la finalidad de generar material lo más claro y preciso posible y que el autor pueda transmitir de manera fácil y sencilla los aspectos claves de su investigación. Además, es importante mencionar que la RMH cuenta con recursos para revisores pares, y así lograr orientarlos en cómo hacer la revisión par asignada.

Un aspecto interesante que refirieron los encuestados son las limitantes actuales para poder colaborar nuevamente con la RMH. Los autores indicaron tres razones de importancia: no saber cómo hacerlo, no tener experiencia y no contar con equipo de trabajo. Por su parte los revisores pares respondieron las mismas razones que los autores. Estos datos son importantes y se deben considerar a futuro para tomar decisiones de mejoras y fortalecimiento desde la $\mathrm{RMH}$, pero en especial desde la formación académica universitaria, en donde se debe hacer un énfasis en asignaturas de la metodología de la investigación. Por el contrario, un estudio realizado por Duracinsky y colaboradores en investigadores europeos reportó que las principales barreras para publicar en las revistas, fue la falta de tiempo en $85 \%$, y dificultades para la redacción de la discusión en $60 \%$. También se reportó que el $87 \%$ investigadores con poca experiencia en publicación, aceptarían apoyo técnico para superar dificultades de publicación. ${ }^{12} \mathrm{Al}$ realizar estos contrastes podemos analizar que las limitaciones son diversas y cambiantes según las culturas donde la importancia de la investigación es totalmente diferente.

Se reconoce que la revisión por pares constituye una actividad fundamental en el proceso de publicación de artículos científicos, sin embargo, aún no consigue el suficiente reconocimiento por parte de la comunidad científica y académica. Quienes participan en esta labor suelen motivarse principalmente por el aprendizaje inherente a la tarea, no obstante, es deseable fortalecer y aumentar continuamente la calidad de las revisiones mediante la evaluación, realimentación y reconocimiento, no solo de los equipos editoriales, sino de otros revisores y de los autores. ${ }^{13}$ Los revisores de revistas biomédicas son actores clave en el ecosistema editorial, ayudan a los autores a mejorar sus manuscritos y asesoran a los editores científicos sobre su decisión sobre la aceptabilidad de los artículos publicados. ${ }^{14} \mathrm{La}$ revisión por pares se define como "la evaluación crítica de los manuscritos enviados a las revistas por parte de expertos que normalmente no forman parte del personal editorial". ${ }^{15}$

El cuerpo editorial es fundamental para dar la orientación a los autores y revisores pares, tal como lo manifestaron los participantes de la investigación al referirse a las respuestas por parte del Consejo Editorial de la RMH, las cuales fueron entre extremadamente claras y claras en un $80 \%$. La gestión orientada a convertir un manuscrito en un artículo científico demanda del intercambio oportuno de criterios e información entre autores, directores de publicaciones científicas, técnicas y especialistas expertos (árbitros) del área de conocimiento o tema que se trate. ${ }^{16}$ También es importante la adopción de un estilo adecuado de comunicación que permita la claridad y entendimiento del trabajo en el ámbito nacional e internacional, este proceso se basa en un conjunto de requisitos que a través de la comunicación entre autor y editor buscan optimizar el artículo científico. ${ }^{17}$ Los editores de revistas deben establecer procedimientos para velar por la calidad de la revista, identificar errores y problemas, detectar tendencias que reflejen un deterioro de la calidad y poner en marcha acciones correctoras según sea necesario. ${ }^{18}$

En este estudio se encontró que un $71.6 \%$ recibió asesoría personalizada para aclarar dudas en relación a la revisión de su manuscrito, destacándose los medios utilizados WhatsApp y vía correo electrónico. El contacto cercano de los editores con los autores es un aspecto fundamental para lograr un proceso editorial exitoso. Por el contrario, en el estudio de D' Souza y colaboradores indicaron que existe una necesidad de hacer el proceso de publicación más amigable, para evitar la brecha entre la comunicación entre el autor y la revista. ${ }^{19}$ En este sentido es fundamental que la RMH mantenga y fortalezca la asesoría personalizada con los autores. La necesidad de asesoría personalizada se hizo evidente en un alto porcentaje del grupo, tanto de autores como revisores y como refiere la investigación realizada por la revista "Investigación en Educación Médica" 
editada por la Facultad de Medicina de la Universidad Nacional Autónoma de México en un grupo de revisores para conocer sus percepciones y experiencias en torno al proceso de revisión por pares, todos los participantes refirieron no haber recibido entrenamiento o capacitación específica para revisar artículos $y$, si bien la experiencia profesional es lo que expresaron como principal estrategia para la evaluación. ${ }^{13}$

Entre las limitaciones de este análisis es que en el reporte de esta encuesta participó menos de la mitad del número de autores y revisores pares invitados, esto pudo deberse a que solo se invitó a los que tuvieron la experiencia en los años 2016-2020, la encuesta fue voluntaria, siendo respondida por los que comprenden la importancia de la retroalimentación de los procesos para la mejora continua. Para profundizar sobre las percepciones de los usuarios, barreras y facilitadores de una participación editorial efectiva, se recomienda realizar una investigación que puede incluir enfoque mixto, cuantitativo y cualitativo, en una muestra representativa de autores, revisores pares y lectores.

Las revistas biomédicas proporcionan un instrumento de inestimable valor para la formación continua, favoreciendo la actualización tanto de los investigadores, revisores y lectores ya sea en el ámbito clínico, académico o de salud pública. Los consejos editoriales asumen el reto de seleccionar a través de los procesos editoriales y difundir de forma eficiente las novedades científicas que nos permiten avanzar en el conocimiento. De hecho, únicamente las revistas biomédicas garantizan el desarrollo íntegro del proceso científico, seleccionando los mejores trabajos originales de investigación para su publicación. ${ }^{20} \mathrm{Sin}$ embargo, persisten los desafíos y uno de ellos es que la investigación biomédica aún no se informa de manera óptima a pesar de la abundancia de pautas de presentación de informes. ${ }^{21} \mathrm{Se}$ puede concluir que los participantes expresaron una percepción positiva y que están satisfechos con el proceso editorial y a su vez están dispuestos a enviar nuevamente un manuscrito o participar como revisores pares. Es necesario impulsar la mejora continua de la RMH a través de la implementación de un sistema de gestión automatizada de revistas científicas como el Open Journal System (OJS) ${ }^{22}$ que mejora los procesos editoriales y a su vez seguir promoviendo el fortalecimiento del ecosistema de la investigación en el gremio médico y sus colaboradores en Honduras.

\section{CONTRIBUCIONES}

EM, JA, participaron en la concepción del estudio. EM, JA y FM trabajaron en el diseño del formulario de recolección de la información. FM recolectó la información. EM, BB, NR, JA, $\mathrm{RD}, \mathrm{ALC}, \mathrm{HG}, \mathrm{EE}$, lideraron la redacción de todo el manuscrito. Todos los autores participaron y contribuyeron en la revisión y aprobación del documento final.

\section{AGRADECIMIENTO}

Se agradece al Ing. Bryan Barrios y a la Señora Marcela Aguilera, Centro Nacional de Educación Médica Continua (CENEMEC), Colegio Médico de Honduras, por la gestión en la recuperación de la información de los formularios en línea completados por los usuarios.

\section{DETALLE DE AUTOR(ES)}

Edna Maradiaga, Médica Salubrista; edjamar3006@yahoo.com Briana Beltran, Médica Epidemióloga; yasmin.beltran@gmail. com

Nora Rodríguez Corea, Médica Pediatra, Master epidemiología; norarmendoz@gmail.com

Rosa María Duarte. Médica Salubrista; duarte2000s|@yahoo. com

Eleonora Espinoza. Médica Salubrista; eleo22@hotmail.com

Flor Mejía. Licenciada en Pedagogía; revmh@colegiomedico.hn Ana Ligia Chinchilla Mejía, Médica Especialista en Ginecología y Obstetricia; ligiachinchilla@yahoo.com.mx

Heriberto Rodríguez Gudiel. Médico Especialista en Ginecología y Obstetricia, con Sub-especialidad en Medicina Materno Fetal; mmfhrg@gmail.com

Melissa Mejía. Médica Especialista en Medicina Física y Rehabilitación; mmejia@colegiomedico.hn

Jackeline Alger. Médica, PhD en Parasitología; jackelinealger@ gmail.com

\section{REFERENCIAS}

1. Carvajal N, Cartagena D. [Editorial] Proceso editorial de la Revista Científica Ciencia Médica: metas cumplidas, nuevos desafíos. Rev Cient Cienc Méd. 2012; 15(2):3-4

2. Gasparyan AY, Yessirkepov M, Voronov AA, Gorin SV, Koroleva AM, Kitas GD. Statement on publication ethics for editors and publishers. J Korean Med Sci. 2016;31(9):1351-4. doi: 10.3346/jkms.2016.31.9.1351.

3. Alger J. [Editorial]. Fortalecimiento y mejora continua de la Revista Médica Hondureña. Rev Med Hondur. 2019;87(1):5.

4. Aquino-Jarquin G, Valencia-Reyes JM, Silva-Carmona A, GranadosRiverón JT. Preimpresiones en biomedicina: ¿alternativa o complemento al modelo tradicional de publicación? [Preprints in biomedicine: alternative or complement to the traditional model of publication?]. Gac Med Mex. 2018;154(1):87-91. Spanish. doi: 10.24875/GMM.17002770.

5. Miyahira J. [Editorial] Criterios de calidad de las revistas científicas. Rev Med Hered. 2008;19(1):1-4

6. Maradiaga E, García C. Análisis bibliométrico de la Revista Médica Hondureña, 1990-2020. Rev Med Hondur. 2021;89(1):45-51.
7. Weber EJ, Katz PP, Waeckerle JF, Callaham ML. Author Perception of Peer Review: Impact of Review Quality and Acceptance on Satisfaction. JAMA. 2002;287(21):2790-2793. doi:10.1001/jama.287.21.2790

8. Pardal-Refoyo JL. Directrices de presentación de informes para los principales tipos de estudio: listas Equator. Revista ORL [Internet]. 2018 [citado 25 octubre 2021];9(4):247-250. Disponible en : https://revistas.usal. es/index.php/2444-7986/article/view/orl.18111

9. Huisman, J., Smits, J. Duration and quality of the peer review process: the author's perspective. Scientometrics [citado el 18-9-2021]113, 633-650 (2017). https://doi.org/10.1007/s11192-017-2310-5

10. Sarigöl E, García D, Scholtes I, Schweitzer F. Cuantificación del efecto de las relaciones editor-autor en los tiempos de manipulación de manuscritos. Cienciometría. 2017;113(1):609-631. doi: 10.1007/s11192-017-2309

11. Pardal-Refoyo JL, Ochoa-Sangrador C. De la investigación a la publicación. El proceso editorial. Rev Soc Otorrinolaringol Castilla Leon Cantab La Rioja. 2013 [citado 18 septiembre 2021];4(9):52-75. Disponible en: https:// revistas.usal.es/index.php/2444-7986/article/view/orl.18111/18455 
12. Duracinsky M, Lalanne $C$, Rous L, Dara AF, Baudoin L, Pellet $C$, et al. Barriers to publishing in biomedical journals perceived by a sample of French researchers: results of the DIAzePAM study. BMC Med Res Methodol [Internet]. 2017 [citado el 24 septiembre 2021];17(96);1-10. Disponible en: https://doi.org/10.1186/s12874-017-0371-z

13. Morales Castillo JD, Fortoul T, Sánchez Mendiola M. La revisión por pares: análisis cualitativo de la experiencia de un grupo de revisores latinoamericanos. Inv Ed Med. 2020 julio-septiembre; 19(35).

14. Glonti K, Boutron I, Moher D, Hren D. Journal editors' perspectives on the roles and tasks of peer reviewers in biomedical journals: a qualitative study. BMJ Open [Internet]. 2019[citado el 20-9-2021];9e:1-10. Disponible en: https://bmjopen.bmj.com/content/9/11/e033421

15. Mack C. The Editorial Review Process. J. Micro/Nanolith. MEMS MOEMS. [Internet] 2015 [citado 29 octubre 2021] jul-sep; 14(3):030101. Disponible en: https://www.researchgate.net/publication/281169824_The_Editorial_ Review_Proces

16. Silva Hernández DDR, Martínez Trujillo N, Del Campo Peña AD, Crosdale Hunt R, Lobaina Baile N, Noa Riverón I. Percepciones de expertos sobre características y habilidades que deben considerarse en la revisión de manuscritos. Rev Cubana Salud Pública. 2019;45(2):e1665.

17. Hernández R. Proceso editorial de una revista científica: cumpliendo con los requisitos de publicación. Rev Peru Psicol Trab Soc. 2015;4(1):77-84.

18. Gómez W. Procesos Editoriales de una Revista Científica. Agora Rev Cient. 2014; 1(1):5-6.

19. D'Souza B, Kulkarni S, Cerejo C. Authors' perspectives on academic publishing: initial observations from a large-scale global survey Sci Ed [Internet] 2018 [citado 24 septiembre 2021];5(1):39-43. Disponible en: https://www.escienceediting.org/journal/view.php?doi=10.6087/kcse.116

20. Alfonso $\mathrm{F}$, Sanchis J. Excelencia editorial y científica de las revistas biomédicas: ¿está todo en el factor de impacto? Arch Cardiol Mex. 2015; 85(4):265-269.

21. Moher D. Reporting guidelines: doing better for readers. BMC Med. 2018; 14;16(1):233. doi: 10.1186/s12916-018-1226-0

22. Alfonso Manzanet JE, Silva Ayçaguer LC. Gestión automatizada en el proceso editorial de una revista científica como demanda inaplazable para favorecer la cultura comunicacional: a pressing demand to facilitate the communicational culture. Educ Med Super [Internet]. 2014[citado 1 diciembre 2021 ];28(1):145-153. Disponible en: http://scielo.sld.cu/scielo. php?script=sci_arttext\&pid=S0864-21412014000100015\&lng=es.
ABSTRACT. Background: The editorial process involves authors, editors, peer reviewers and readers, who contribute to the quality of biomedical publications. Objective: To evaluate the results of a satisfaction survey aimed at authors and peer reviewers who participated in the editorial process of the Revista Médica Hondureña (RMH), period 2016-2020. Methodology: Retrospective analysis of forms completed online by authors and peer reviewers, invited to participate voluntarily by email during December 2020-July 2021. The survey included 16 questions that recorded characteristics of the editorial process and suggestions for improvement. Satisfaction was classified into 5 categories, from very satisfied to very dissatisfied. The results are presented as frequencies and percentages of the variables studied. Results: We analyzed 53 surveys, completed by $47(88.7 \%)$ authors and $6(11.3 \%)$. The $94.3 \%$ (50) described as clear instructions for authors, $77.4 \%$ (41) indicated having received a timely response, $71.7 \%$ (38) received personalized advice, $90.5 \%$ (48) described as very likely/likely their willingness to send articles or collaborate as a peer reviewer; some limitations to participate were lack of time and inexperience. The $69.8 \%$ (37) expressed very satisfied/satisfied compared to $17.0 \%$ (9) who expressed dissatisfaction with the editorial process. Discussion: In this limited sample of users, more than $2 / 3$ expressed a positive perception and satisfaction with the editorial process. It is necessary to promote the continuous improvement of the $\mathrm{RMH}$ and promote the strengthening of the research ecosystem in the medical guild and collaborators in Honduras.

Keywords. Authorship and co-authorship in scientific publications; Editorial polices; Peer review; Personal satisfaction; Scholarly communication. 